\title{
Applying Explanation-based Learning to Control and Speeding-up Natural Language Generation
}

\author{
Günter Neumann \\ DFKI GmbH \\ Stuhlsatzenhausweg 3 \\ 66123 Saarbrücken, Germany \\ neumann@dfki.uni-sb.de
}

\begin{abstract}
This paper presents a method for the automatic extraction of subgrammars to control and speeding-up natural language generation NLG. The method is based on explanation-based learning EBL. The main advantage for the proposed new method for NLG is that the complexity of the grammatical decision making process during NLG can be vastly reduced, because the EBL method supports the adaption of a NLG system to a particular use of a language.
\end{abstract}

\section{Introduction}

In recent years, a Machine Learning technique known as Explanation-based Learning EBL (Mitchell, Keller, and Kedar-Cabelli, 1986; van Harmelen and Bundy, 1988; Minton et al., 1989) has successfully been applied to control and speeding-up natural language parsing (Rayner, 1988; Samuelsson and Rayner, 1991; Neumann, 1994a; Samuelsson, 1994; Srinivas and Joshi, 1995; Rayner and Carter, 1996). The core idea of EBL is to transform the derivations (or explanations) computed by a problem solver (e.g., a parser) to some generalized and compact forms, which can be used very efficiently for solving similar problems in the future. EBL has primarily been used for parsing to automatically specialize a given source grammar to a specific domain. In that case, EBL is used as a method for adapting a general grammar and/or parser to the sub-language defined by a suitable training corpus (Rayner and Carter, 1996).

A specialized grammar can be seen as describing a domain-specific set of prototypical constructions. Therefore, the EBL approach is also very interesting for natural language generation (NLG). Informally, NLG is the production of a natural language text from computer-internal representation of information, where NLG can be seen as a complex - potentially cascaded--decision making process. Commonly, a NLG system is decomposed into two major components, viz. the strategic component which decides 'what to say' and the tactical component which decides 'how to say' the result of the strategic component. The input of the tactical component is basically a semantic representation computed by the strategic component. Using a lexicon and a grammar, its main task is the computation of potentially all possible strings associated with a semantic input. Now, in the same sense as EBL is used in parsing as a means to control the range of possible strings as well as their degree of ambiguity, it can also be used for the tactical component to control the range of possible semantic input and their degree of paraphrases.

In this paper, we present a novel method for the automatic extraction of subgrammars for the control and speeding-up of natural language generation. Its main advantage for NLG is that the complexity of the (linguistically oriented) decision making process during natural language generation can be vastly reduced, because the EBL method supports adaption of a NLG system to a particular language use. The core properties of this new method are:

- prototypical occuring grammatical constructions can automatically be extracted;

- generation of these constructions is vastly sped up using simple but efficient mechanisms;

- the new method supports partial matching, in the sense that new semantic input need not be completely covered by previously trained examples;

- it can easily be integrated with recently developed chart-based generators as described in, 
e.g., (Neumann, 1994b; Kay, 1996; Shemtov, 1996).

The method has been completely implemented and tested with a broad-coverage HPSG-based grammar for English (see sec. 5 for more details).

\section{Foundations}

The main focus of this paper is tactical generation, i.e., the mapping of structures (usually representing semantic information eventually decorated with some functional features) to strings using a lexicon and a grammar. Thus stated, we view tactical generation as the inverse process of parsing. Informally, EBL can be considered as an intelligent storage unit of example-based generalized parts of the grammatical search space determined via training by the tactical generator. ${ }^{1}$ Processing of similar new input is then reduced to simple lookup and matching operations, which circumvent re-computation of this already known search space.

We concentrate on constraint-based grammar formalism following a sign-based approach considering linguistic objects (i.e., words and phrases) as utterance-meaning associations (Pollard and Sag, 1994). Thus viewed, a grammar is a formal statement of the relation between utterances in a natural language and representations of their meanings in some logical or other artificial language, where such representations are usually called logical forms (Shieber, 1993). The result of the tactical generator is a feature structure (or a set of such structures in the case of multiple paraphrases) containing among others the input logical form, the computed string, and a representation of the derivation.

In our current implementation we are using TDL, a typed feature-based language and inference system for constraint-based grammars (Krieger and Schäfer, 1994). TDL allows the user to define hierarchicallyordered types consisting of type and feature constraints. As shown later, a systematic use of type information leads to a very compact representation of the extracted data and supports an elegant but efficient generalization step.

We are adapting a "flat" representation of logical forms as described in (Kay, 1996; Copestake et al., 1996). This is a minimally structured, but descriptively adequate means to represent semantic information, which allows for various types of under-/overspecification, facilitates generation and the specification of semantic transfer equivalences

\footnotetext{
${ }^{1}$ In case a reversible grammar is used the parser can even be used for processing the training corpus.
}

used for machine translation (Copestake et al., 1996; Shemtov, 1996). ${ }^{2}$

Informally, a flat representation is obtained by the use of extra variables which explicitly represent the relationship between the entities of a logical form and scope information. In our current system we are using the framework called minimal recursion semantics (MRS) described in (Copestake et al., 1996). Using their typed feature structure notation figure 1 displays a possible MRS of the string "Sandy gives a chair to Kim" (abbreviated where convenient).

The value of the feature LISZT is actually treated like a set, i.e., the relative order of the elements is immaterial. The feature HANDEL is used to represent scope information, and INDEX plays much the same role as a lambda variable in conventional representations (for more details see (Copestake et al., 1996)).

\section{Overview of the method}

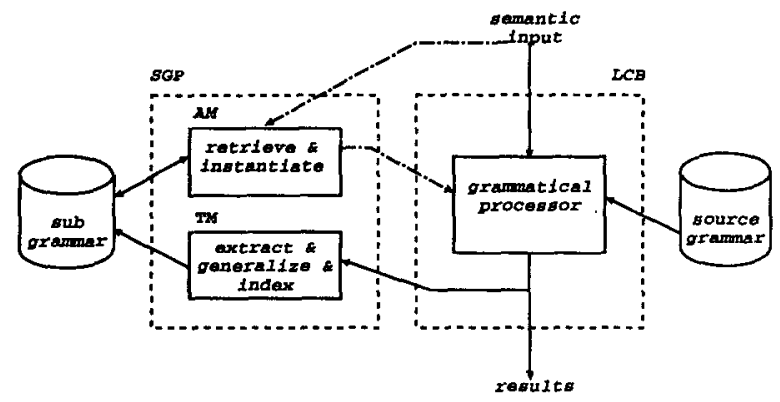

Figure 3: A blueprint of the architecture.

The above figure displays the overall architecture of the EBL learning method. The right-hand part of the diagram shows the linguistic competence base (LCB) and the left the EBL-based subgrammar processing component (SGP).

LCB corresponds to the tactical component of a general natural language generation system NLG. In this paper we assume that the strategic component of the NLG has already computed the MRS representation of the information of an underlying computer program. SGP consists of a training module $\mathrm{TM}$, an application module $\mathrm{AM}$, and the subgram-

\footnotetext{
${ }^{2}$ But note, our approach does not depend on a flat representation of logical forms. However, in the case of conventional representation form, the mechanisms for indexing the trained structures would require more complex abstract data types (see sec. 4 for more details).
} 


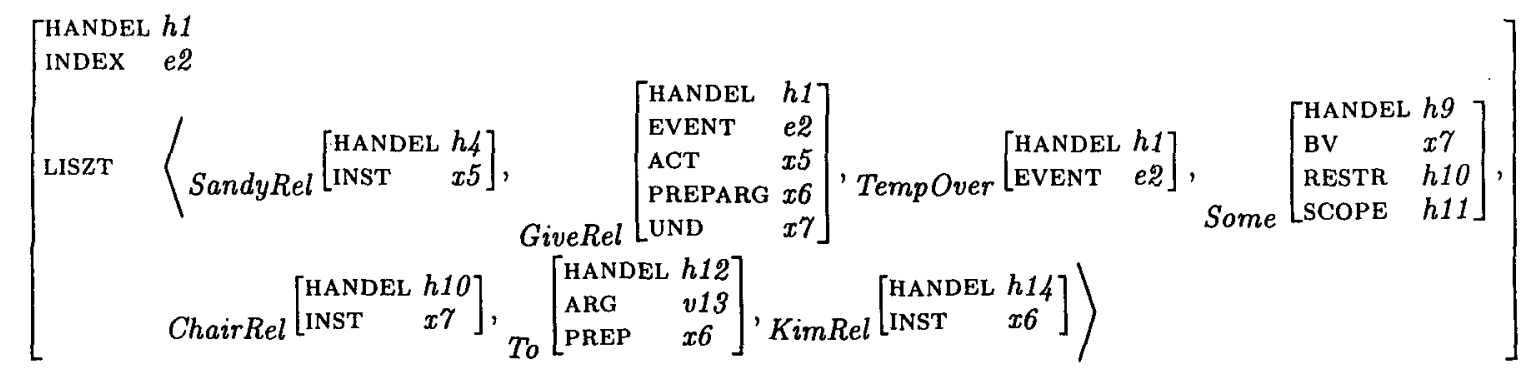

Figure 1: The MRS of the string "Sandy gives a chair to Kim"

$$
\left[\begin{array}{l}
\text { Liszt } \\
\langle\text { SandyRel } \\
\text { ChairRel }
\end{array}\right.
$$

Figure 2: The generalized MRS of the string "Sandy gives a chair to Kim"

mar, automatically determined by TM and applied by AM.

Briefly, the flow of control is as follows: During the training phase of the system, a new logical form $m r s$ is given as input to the LCB. After grammatical processing, the resulting feature structure $f s(\mathrm{mrs})$ (i.e., a feature structure that contains among others the input MRS, the computed string and a representation of the derivation tree) is passed to TM. TM extracts and generalizes the derivation tree of $f s(m r s)$, which we call the template templ(mrs) of $f s(m r s)$. templ(mrs) is then stored in a decision tree, where indices are computed from the MRS found under the root of templ(mrs). During the application phase, a new semantic input $m r s^{\prime}$ is used for the retrieval of the decision tree. If a candidate template can be found and successfully instantiated, the resulting feature structure $f s\left(m r s^{\prime}\right)$ constitutes the generation result of $m r s^{\prime}$.

Thus described, the approach seems to facilitate only exact retrieval and matching of a new semantic input. However, before we describe how partial matching is realized, we will demonstrate in more detail the exact matching strategy using the example MRS shown in figure 1 .

Training phase The training module TM starts right after the resulting feature structure $f s$ for the input MRS $m r s$ has been computed. In the first phase, TM extracts and generalizes the derivation tree of $f s$, called the template of $f s$. Each node of the template contains the rule name used in the corresponding derivation step and a generalization of the local MRS. A generalized MRS is the abstraction of the LISZT value of a MRS where each element only contains the (lexical semantic) type and HANDEL information (the HANDEL information is used for directing lexical choice (see below)).

In our example $m r s$, figure 2 displays the generalized MRS $m r s_{g}$. For convenience, we will use the more compact notation:

\section{\{(SandyRel h4), (GiveRel h1),} (TempOver h1), (Some h9),

(ChairRel h10), (To h12), (KimRel h14)\}

Using this notation, figure 4 (see next page) displays the template templ(mrs) obtained from $f s$. Note that it memorizes not only the rule application structure of a successful process but also the way the grammar mutually relates the compositional parts of the input MRS.

In the next step of the training module TM, the generalized MRS $m r s_{g}$ information of the root node of templ(mrs) is used for building up an index in a decision tree. Remember that the relative order of the elements of a MRS is immaterial. For that reason, the elements of $m r s_{g}$ are alphabetically ordered, so that we can treat it as a sequence when used as a new index in the decision tree.

The alphabetic ordering has two advantages. Firstly, we can store different templates under a common prefix, which allows for efficient storage and retrieval. Secondly, it allows for a simple efficient treatment of MRS as sets during the retrieval phase of the application phase. 


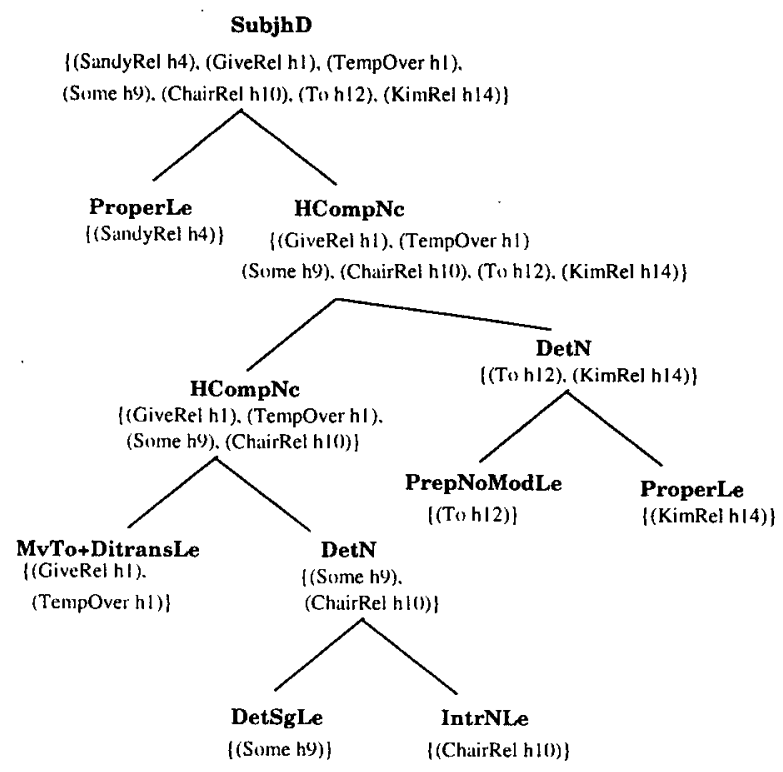

Figure 4: The template templ(mrs). Rule names are in bold.

Application phase The application module AM basically performs the following steps:

1. Retrieval: For a new MRS $m r s^{\prime}$ we first construct the alphabetically sorted generalized MRS $m r s_{g}^{\prime}$. $m r s_{g}^{\prime}$ is then used as a path description for traversing the decision tree. For reasons we will explain soon, traversal is directed by type subsumption. Traversal is successful if $m r s_{g}^{\prime}$ has been completely processed and if the end node in the decision tree contains a template. Note that because of the alphabetic ordering, the relative order of the elements of new input $m r s^{\prime}$ is immaterial.

2. Expansion: A successfully retrieved template templ is expanded by deterministically applying the rules denoted by the non-terminal elements from the top downwards in the order specified by templ. In some sense, expansion just re-plays the derivation obtained in the past. This will result in a grammatically fully expanded feature structure, where only lexical specific information is still missing. But note that through structure sharing the terminal elements will already be constrained by syntactic information. ${ }^{3}$

\footnotetext{
${ }^{3}$ It is possible to perform the expansion step off-line as early as the training phase, in which case the application phase can be sped up, however at the price of more memory being taken up.
}

3. Lexical lookup: From each terminal element of the unexpanded template templ the type and HANDEL information is used to select the corresponding element from the input MRS $m r s^{\prime}$ (note that in general the MRS elements of the $m r s^{\prime}$ are much more constrained than their corresponding elements in the generalized MRS $m r s_{g}^{\prime}$ ). The chosen input MRS element is then used for performing lexical lookup, where lexical elements are indexed by their relation name. In general this will lead to a set of lexical candidates.

4. Lexical instantiation: In the last step of the application phase, the set of selected lexical elements is unified with the constraints of the terminal elements in the order specified by the terminal yield. We also call this step terminalmatching. In our current system terminalmatching is performed from left to right. Since the ordering of the terminal yield is given by the template, it is also possible to follow other selection strategies, e.g., a semantic head-driven strategy, which could lead to more efficient terminal-matching, because the head element is supposed to provide selectional restriction information for its dependents.

A template together with its corresponding index describes all sentences of the language that share the same derivation and whose MRS are consistent with that of the index. Furthermore, the index and the MRS of a template together define a normalization for the permutation of the elements of a new input MRS. The proposed EBL method guarantees soundness because retaining and applying the original derivation in a template enforces the full constraints of the original grammar.

Achieving more generality So far, the application phase will only be able to re-use templates for a semantic input which has the same semantic type information. However, it is possible to achieve more generality, if we apply a further abstraction step on a generalized MRS. This is simply achieved by selecting a supertype of a MRS element instead of the given specialized type.

The type abstraction step is based on the standard assumption that the word-specific lexical semantic types can be grouped into classes representing morpho-syntactic paradigms. These classes define the upper bounds for the abstraction process. In our current system, these upper bounds are directly used as the supertypes to be considered during the type abstraction step. More precisely, for each element $x$ of a generalized MRS $m r s_{g}$ it is checked 
whether its type $T_{x}$ is subsumed by an upper bound $T_{s}$ (we assume disjoint sets). Only if this is the case, $T_{s}$ replaces $T_{x}$ in $m r s_{g}{ }^{4}$ Applying this type abstraction strategy on the MRS of figure 1, we obtain:

$\{($ Named h4), (ActUndPrep h1), (TempOver h1), (Some h9), (RegNom h10), (To h12), (Named h14)\}

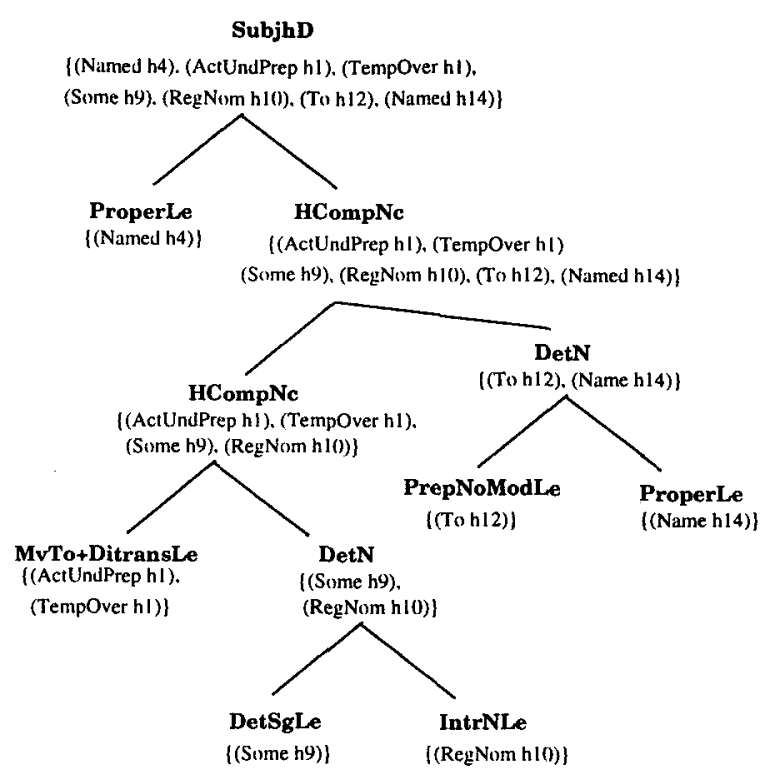

Figure 5: The more generalized derivation tree $d t_{g}$ of $d t$.

where e.g., NAMED is the common supertype of SANDYREL and KIMREL, and ACTUNDPREP is the supertype of GiveREL. Figure 5 shows the template templ $l_{g}$ obtained from $f s$ using the more general MRS information. Note, that the MRS of the root node is used for building up an index in the decision tree.

Now, if retrieval of the decision tree is directed by type subsumption, the same template can be retrieved and potentially instantiated for a wider range of new MRS input, namely for those which are type compatible wrt. subsumption relation. Thus, the template templ $_{g}$ can now be used to generate, e.g., the string "Kim gives a table to Peter", as well as the string "Noam donates a book to Peter".

However, it will not be able to generate a sentence like "A man gives a book to Kim", since the retrieval

\footnotetext{
${ }^{4}$ Of course, if a very fine-grained lexical semantic type hierarchy is defined then a more careful selection would be possible to obtained different degrees of type abstraction and to achieve a more domain-sensitive determination of the subgrammars. However, more complex type abstraction strategies are then needed which would be able to find appropriate supertypes automatically.
}

phase will already fail. In the next section, we will show how to overcome even this kind of restriction.

\section{Partial Matching}

The core idea behind partial matching is that in case an exact match of an input MRS fails we want at least as many subparts as possible to be instantiated. Since the instantiated template of a MRS subpart corresponds to a phrasal sign, we also call it a phrasal template. For example, assuming that the training phase has only to be performed for the example in figure 1, then for the MRS of "A man gives a book to Kim", a partial match would generate the strings "a man" and "gives a book to Kim". 5 The instantiated phrasal templates are then combined by the tactical component to produce larger units (if possible, see below).

Extended training phase The training module is adapted as follows: Starting from a template templ obtained for the training example in the manner described above, we extract recursively all possible subtrees templs also called phrasal templates. Next, each phrasal template is inserted in the decision tree in the way described above.

It is possible to direct the subtree extraction process with the application of filters, which are applied to the whole remaining subtree in each recursive step. By using these filters it is possible to restrict the range of structural properties of candidate phrasal templates (e.g., extract only saturated NPs, or subtrees having at least two daughters, or subtrees which have no immediate recursive structures). These filters serve the same means as the "chunking criteria" described in (Rayner and Carter, 1996).

During the training phase it is recognized for each phrasal template templ $l_{s}$ whether the decision tree already contains a path pointing to a previously extracted and already stored phrasal template templ $_{s}^{\prime}$, such that templ $=$ templ $_{s}^{\prime}$. In that case, templ $l_{s}$ is not inserted and the recursion stops at that branch.

Extended application phase For the application module, only the retrieval operation of the decision tree need be adapted.

Remember that the input of the retrieval operation is the sorted generalized MRS $m r s_{g}$ of the input MRS mrs. Therefore, $m r s_{g}$ can be handled like a sequence. The task of the retrieval operation in the case of a partial match is now to potentially find all subsequences of $m r s_{g}$ which lead to a template.

\footnotetext{
${ }^{5}$ If we would allow for an exhaustive partial match (see below) then the strings "a book" and "Kim" would additionally be generated.
} 
In case of exact matching strategy, the decision tree must be visited only once for a new input. In the case of partial matching, however, the decision tree describes only possible prefixes for a new input. Hence, we have to recursively repeat retrieval of the decision tree as long as the remaining suffix is not empty. In other words, the decision tree is now a finite representation of an infinite structure, because implicitly, each endpoint of an index bears a pointer to the root of the decision tree.

Assuming that the following template/index pairs have been inserted into the decision tree: $\left\langle a b, t_{1}\right\rangle$, $\left\langle a b c d, t_{2}\right\rangle,\left\langle b c d, t_{3}\right\rangle$. Then retrieval using the path $a b c d$ will return all three templates, retrieval using $a a b b c d$ will return template $t_{1}$ and $t_{3}$, and $a b c$ will only return $t_{1}{ }^{6}$

Interleaving with normal processing Our EBL method can easily be integrated with normal processing, because each instantiated template can be used directly as an already found sub-solution. In case of an agenda-driven chart generator of the kind described in (Neumann, 1994a; Kay, 1996), an instantiated template can be directly added as a passive edge to the generator's agenda. If passive edges with a wider span are given higher priority than those with a smaller span, the tactical generator would try to combine the largest derivations before smaller ones, i.e., it would prefer those structures determined by EBL.

\section{Implementation}

The EBL method just described has been fully implemented and tested with a broad coverage HPSGbased English grammar including more than 2000 fully specified lexical entries. ${ }^{7}$ The TDL grammar formalism is very powerful, supporting distributed disjunction, full negation, as well as full boolean type logic.

In our current system, an efficient chart-based bidirectional parser is used for performing the training phase. During training, the user can interactively select which of the parser's readings should be considered by the EBL module. In this way the user can control which sort of structural ambiguities should be avoided because they are known to cause misunderstandings. For interleaving the EBL application phase with normal processing a first pro-

\footnotetext{
${ }^{6}$ It is possible to parameterize our system to perform an exhaustive or a non-exhaustive strategy. In the non-exhaustive mode, the longest matching prefixes are preferred.

${ }^{7}$ This grammar has been developed at CSLI, Stanford, and kindly be provided to the author.
}

totype of a chart generator has been implemented using the same grammar as used for parsing.

First tests has been carried out using a small test set of 179 sentences. Currently, a parser is used for processing the test set during training. Generation of the extracted templates is performed solely by the EBL application phase (i.e., we did not considered integration of EBL and chart generation). The application phase is very efficient. The average processing time for indexing and instantiation of a sentence level template (determined through parsing) of an input MRS is approximately one second. ${ }^{8}$ Compared to parsing the corresponding string the factor of speed up is between 10 to 20 . A closer look to the four basic EBL-generation steps: indexing, instantiation, lexical lookup, and terminal matching showed that the latter is the most expensive one (up to $70 \%$ of computing time). The main reasons are that 1.) lexical lookup often returns several lexical readings for an MRS element (which introduces lexical non-determinism) and 2.) the lexical elements introduce most of the disjunctive constraints which makes unification very complex. Currently, terminal matching is performed left to right. However, we hope to increase the efficiency of this step by using head-oriented strategies, since this might help to re-solve disjunctive constraints as early as possible.

\section{Discussion}

The only other approach I am aware of which also considers EBL for NLG is (Samuelsson, 1995a; Samuelsson, 1995b). However, he focuses on the compilation of a logic grammar using LR-compiling techniques, where EBL-related methods are used to optimize the compiled LR tables, in order to avoid spurious non-determinisms during normal generation. He considers neither the extraction of a specialized grammar for supporting controlled language generation, nor strong integration with the normal generator.

However, these properties are very important for achieving high applicability. Automatic grammar extraction is worthwhile because it can be used to support the definition of a controlled domain-specific language use on the basis of training with a general source grammar. Furthermore, in case exact matching is requested only the application module is needed for processing the subgrammar. In case of normal processing, our EBL method serves as a speed-up mechanism for those structures which have

\footnotetext{
${ }^{8}$ EBL-based generation of all possible templates of an input MRS is less than 2 seconds. The tests have been performed using a Sun UltraSparc.
} 
"actually been used or uttered". However, completeness is preserved.

We view generation systems which are based on "canned text" and linguistically-based systems simply as two endpoints of a contiguous scale of possible system architectures (see also (Dale et al., 1994)). Thus viewed, our approach is directed towards the automatic creation of application-specific generation systems.

\section{Conclusion and Future Directions}

We have presented a method of automatic extraction of subgrammars for controlling and speeding up natural language generation (NLG). The method is based on explanation-based learning (EBL), which has already been successfully applied for parsing. We showed how the method can be used to train a system to a specific use of grammatical and lexical usage.

We already have implemented a similar EBL method for parsing, which supports on-line learning as well as statistical-based management of extracted data. In the future we plan to combine EBLbased generation and parsing to one uniform EBL approach usable for high-level performance strategies which are based on a strict interleaving of parsing and generation (cf. (Neumann and van Noord, 1994; Neumann, 1994a)).

\section{Acknowledgement}

The research underlying this paper was supported by a research grant from the German Bundesministerium für Bildung, Wissenschaft, Forschung und Technologie $(B M B+F)$ to the DFKI project PARADIME FKZ ITW 9704.

I would like to thank the HPSG people from CSLI, Stanford for their kind support and for providing the HPSG-based English grammar. In particular I want to thank Dan Flickinger and Ivan Sag. Many thanks also to Walter Kasper for fruitful discussions.

\section{References}

Copestake, A., D. Flickinger, R. Malouf, S. Riehemann, and I. Sag. 1996. Translation using minimal recursion semantics. In Proceedings, 6th International Conference on Theoretical and Methodological Issues in Machine Translation.

Dale, R., W. Finkler, R. Kittredge, N. Lenke, G. Neumann, C. Peters, and M. Stede. 1994. Report from working group 2: Lexicalization and architecture. In W. Hoeppner, H. Horacek, and J. Moore, editors, Principles of Natural Language
Generation, Dagstuhl-Seminar-Report; 93. Schloß Dagstuhl, Saarland, Germany, Europe, pages 3039.

Kay, M. 1996. Chart generation. In 34th Annual Meeting of the Association for Computational Linguistics, Santa Cruz, Ca.

Krieger, Hans-Ulrich and Ulrich Schäfer. 1994. $\mathcal{T D} \mathcal{L}$ - a type description language for constraintbased grammars. In Proceedings of the 15th International Conference on Computational Linguistics, COLING-94, pages 893-899.

Minton, S., J. G. Carbonell, C. A. Knoblock, D. R.Kuokka, O. Etzioni, and Y.Gi. 1989. Explanation-based learning: A problem solving perspective. Artificial Intelligence, 40:63-115.

Mitchell, T., R. Keller, and S. Kedar-Cabelli. 1986. Explanation-based generalization: a unifying view. Machine Learning, 1:47-80.

Neumann, G. 1994a. Application of explanationbased learning for efficient processing of constraint based grammars. In Proceedings of the Tenth IEEE Conference on Artificial Intelligence for Applications, pages 208-215, San Antonio, Texas, March.

Neumann, G. 1994b. A Uniform Computational Model for Natural Language Parsing and Generation. Ph.D. thesis, Universität des Saarlandes, Germany, Europe, November.

Neumann, G. and G. van Noord. 1994. Reversibility and self-monitoring in natural language generation. In Tomek Strzalkowski, editor, Reversible Grammar in Natural Language Processing. Kluwer, pages 59-96.

Pollard, C. and I. M. Sag. 1994. Head-Driven Phrase Structure Grammar. Center for the Study of Language and Information Stanford.

Rayner, M. 1988. Applying explanation-based generalization to natural language processing. In Proceedings of the International Conference on Fifth Generation Computer Systems, Tokyo.

Rayner, M. and D. Carter. 1996. Fast parsing using pruning and grammar specialization. In 34 th Annual Meeting of the Association for Computational Linguistics, Morristown, New Jersey.

Samuelsson, C. 1994. Fast Natural-Language Parsing Using Explanation-Based Learning. Ph.D. thesis, Swedish Institute of Computer Science, Kista, Sweden, Europe.

Samuelsson, C. 1995a. An efficient algorithm for surface generation. In Proceedings of the 14 th International Joint Conference on Artificial Intelligence, pages 1414-1419, Montreal, Canada. 
Samuelsson, C. 1995b. Example-based optimization of surface-generation tables. In Proceedings of Recent Advances in Natural Language Processing, Velingrad, Bulgaria, Europe.

Samuelsson, C. and M. Rayner. 1991. Quantitative evaluation of explanation-based learning as an optimization tool for a large-scale natural language system. In IJCAI-91, pages 609-615, Sydney, Australia.

Shemtov, H. 1996. Generation of Paraphrases from Ambiguous Logical Forms. In Proceedings of the 16th International Conference on Computational Linguistics (COLING), pages 919-924, Kopenhagen, Denmark, Europe.

Shieber, S. M. 1993. The problem of logical-form equivalence. Computational Linguistics, 19:179190.

Srinivas, B. and A. Joshi. 1995. Some novel applications of explanation-based learning to parsing lexicalized tree-adjoining grammars. In $33 t h$ Annual Meeting of the Association for Computational Linguistics, Cambridge, MA.

van Harmelen, F. and A. Bundy. 1988. Explanationbased generalization $=$ partial evaluation. Artificial Intelligence, 36:401-412. 